Bull. Korean Math. Soc. 51 (2014), No. 3, pp. 789-801

http://dx.doi.org/10.4134/BKMS.2014.51.3.789

\title{
GROUND STATE SOLUTIONS OF NON-RESONANT COOPERATIVE ELLIPTIC SYSTEMS WITH SUPERLINEAR TERMS
}

\author{
Guanwei Chen
}

\begin{abstract}
In this paper, we study the existence of ground state solutions for a class of non-resonant cooperative elliptic systems by a variant weak linking theorem. Here the classical Ambrosetti-Rabinowitz superquadratic condition is replaced by a general super quadratic condition.
\end{abstract}

\section{Introduction and main result}

In this paper, we consider the following cooperative elliptic system

$$
\begin{cases}-\triangle u=\xi u+f(x, u, v) & \text { in } \Omega, \\ -\triangle v=\zeta v+g(x, u, v) & \text { in } \Omega, \\ u=v=0 & \text { on } \partial \Omega,\end{cases}
$$

where $\Omega$ is an bounded smooth domain in $\mathbb{R}^{N}$ and $\xi, \zeta \in \mathbb{R}$. The nonlinearities $(f, g)$ are the gradient of some function, that is, there exists a function $F(x, U) \in C^{1}\left(\bar{\Omega} \times \mathbb{R}^{2}, \mathbb{R}\right)$ such that $\nabla F(x, U)=(f, g)$, the so-called cooperative case.

We are interested in the case where $\sigma\left(A^{*}\right) \cap \sigma(-\triangle)=\emptyset$ holds, that is, the non-resonant case. Here,

$$
A^{*}=\left(\begin{array}{cc}
\xi & 0 \\
0 & \zeta
\end{array}\right), \quad \sigma\left(A^{*}\right)=\{\xi, \zeta\}
$$

denotes the spectrum of the matrix $A^{*}$ and $\sigma(-\triangle)=\left\{\lambda_{k}: k=1,2, \ldots\right.$ and $\left.0<\lambda_{1}<\lambda_{2}<\cdots\right\}$ denotes the eigenvalues of the Laplacian on $\Omega$ with zero boundary condition.

Let $L_{1}:=-\triangle-\xi$ and $L_{2}:=-\triangle-\zeta$. We assume

$\left(\mathbf{L}_{1}\right) \sup \left(\sigma\left(L_{i}\right) \cap(-\infty, 0)\right)<0<\inf \left(\sigma\left(L_{i}\right) \cap(0, \infty)\right)$, where $i=1,2$.

Received April 9, 2013.

2010 Mathematics Subject Classification. 35J20, 35J55, 35J65.

Key words and phrases. non-resonant cooperative elliptic systems, ground state solutions; superlinear, variant weak linking theorem.

Research supported by the NSFC (Tianyuan Fund for Mathematics, Grant No. 11326113) and the Key Project of Natural Science Foundation of Educational Committee of Henan Province of China (Grant No. 13A110015). 
Remark 1.1. Note that assumption $\left(L_{1}\right)$ implies that $\sigma\left(A^{*}\right) \cap \sigma(-\triangle)=\emptyset$ with $\lambda_{k}<\xi<\lambda_{k+1}$ and $\lambda_{m}<\zeta<\lambda_{m+1}$ for some positive integers $k$ and $m$, that is, the non-resonant case.

Let $\widetilde{F}(x, U):=\frac{1}{2}(\nabla F(x, U), U)-F(x, U)$. We assume that

$\left(\mathbf{S}_{1}\right)|\nabla F(x, U)|=o(|U|)$ as $|U| \rightarrow 0$ uniformly in $x$.

$\left(\mathbf{S}_{\mathbf{2}}\right) \frac{F(x, U)}{|U|^{2}} \rightarrow \infty$ as $|U| \rightarrow \infty$ uniformly in $x$.

$\left(\mathbf{S}_{3}\right) \widetilde{F}(x, U)>0$ if $U \neq 0, F(x, U) \geq 0$ for all $(x, U) \in \Omega \times \mathbb{R}^{2}$.

$\left(\mathbf{S}_{4}\right)$ There exist $c_{0}, r_{0}>0$ and $\sigma>\max \{1, N / 2\}$ such that $\frac{|\nabla F(x, U)|^{\sigma}}{|U|^{\sigma}} \leq$ $c_{0} \widetilde{F}(x, U)$ if $|U| \geq r_{0}$. Here, $|\cdot|$ and $(\cdot, \cdot)$ denote, respectively, the usual norm and inner product in $\mathbb{R}^{2}$.

In this paper, we study the existence of ground state solutions of (1.1) by a variant generalized weak linking theorem for strongly indefinite problem developed by Schechter and Zou [15], that is, nontrivial solutions with least energy of the action functional of (1.1). Now, our main result reads as follows:

Theorem 1.1. If $\left(L_{1}\right)$ and $\left(S_{1}\right)-\left(S_{4}\right)$ hold, then there exists a ground state solution of (1.1).

Assume that there exists a constant $\mu>2$ such that

$$
0<\mu F(x, U) \leq(\nabla F(x, U), U), \quad x \in \Omega \backslash\{0\},
$$

which is now known as Ambrosetti-Rabinowitz superlinear condition. As we all know that condition (1.2) will make our proofs more easier. But we use a more general superquadratic condition. As is shown in next example, our assumptions are reasonable and there are cases in which the condition (1.2) is not satisfied.

\section{Example 1.1. Let}

$$
F(x, U)=g(x)\left(|U|^{p}+(p-2)|U|^{p-\varepsilon} \sin ^{2}\left(|U|^{\varepsilon} / \varepsilon\right)\right),
$$

where $p>2,0<\varepsilon<p-2$ if $N=1,2$ and $0<\varepsilon<p+N-N p / 2$ if $N \geq 3$ and $g(x)>0$ is continuous. Note that

$$
\begin{aligned}
\nabla F(x, U)=g(x) U[ & (p-2)(p-\varepsilon)|U|^{p-\varepsilon-2} \sin ^{2}\left(\frac{|U|^{\varepsilon}}{\varepsilon}\right) \\
& \left.+\left(p+(p-2) \sin \left(\frac{2 \mid U^{\varepsilon}}{\varepsilon}\right)\right)|U|^{p-2}\right] .
\end{aligned}
$$

It is not hard to check that $F(x, U)$ satisfies $\left(S_{1}\right)-\left(S_{4}\right)$. But it does not satisfy the condition (1.2).

We should mention that system $(1.1)$ is called resonant if $\sigma\left(A^{*}\right) \cap \sigma(-\triangle) \neq \emptyset$. If $\xi=\zeta=\lambda_{k} \in \sigma(-\Delta), f(x, u, v)=\bar{f}(x, u), g(x, u, v)=\bar{g}(x, v)$ and $\bar{f} \equiv \bar{g}$ on $\bar{\Omega} \times \mathbb{R}$, then (1.1) reduces to the following single elliptic equation

$$
\begin{cases}-\triangle u=\lambda_{k} u+\bar{f}(x, u) & \text { in } \Omega, \\ u=0 & \text { on } \partial \Omega .\end{cases}
$$


The authors $[5,16]$ have considered the strongly resonant single elliptic equation (1.3) with odd nonlinearities and obtained a finite number of solutions. Li and Zou [8] investigated (1.3) by using the Morse theory. We should mention that the some authors $[13,14]$ have considered the following elliptic systems

$$
\left\{\begin{array}{c}
-\triangle u+V(x) u=f(v) \quad \text { in } \Omega, \\
-\triangle v+V(x) v=g(u) \quad \text { in } \Omega, \\
u, v \in H_{0}^{1}(\Omega),
\end{array}\right.
$$

which can be formulated as a variational problem associated with an indefinite energy functional to find solutions of such a system under suitable growth assumptions on $f$ and $g$. The authors [13, 14] also studied ground states.

The system (1.1) has been studied by many authors under asymptotically linear or sublinear assumptions on nonlinearities, see $[3,9,10,11,19,20,21]$. In [3], the variational structure was established and several existence results were obtained by minimax techniques under a condition which was called nonquadraticity at infinity. Ma [9] established the existence of infinitely many solutions for (1.1) with odd nonlinearities by the minimax techniques. Ma [10] and Zou [21] established the existence and multiplicity of solutions for (1.1) via the computations of the critical groups and the Morse theory. By using a penalization technique and the Morse theory, Pomponio [11] established the existence and multiplicity of solutions of (1.1). However, very little is known about the existence of infinitely many solutions for resonant single elliptic equation and elliptic systems (both cooperative and noncooperative). Zou [19] considered (1.1) and, by using the methods used in [5], obtained infinitely many solutions under the oddness and boundedness assumptions on the nonlinearities. Zou [20] proved that (1.1) has infinitely many solutions under the oddness assumption and some growth assumptions near $U=0$. Recently, if $F(x, U)$ is even in $U$, Chen and $\mathrm{Ma}$ [1] obtained the existence of infinitely many solutions for (1.1) with sublinear or superlinear terms by using two variant fountain theorems. In the whole space $\mathbb{R}^{N}$, Chen and Ma [2] obtained the existence of nontrivial homoclinic solutions for (1.1) with $\Omega=\mathbb{R}^{N}$. For related topics, including noncooperative elliptic systems, we refer the readers to $[4,6,7,12,17,22]$ and references cited therein.

The rest of the present paper is organized as follows. In Section 2, we give some preliminary lemmas, which are useful in the proof of our main result. In Section 3, we give the detailed proof of our main result.

\section{Preliminaries}

Here and in what follows, we use $\|\cdot\|_{p}$ to denote the norm of $L^{p}(\Omega) \times L^{p}(\Omega)$, $p \geq 1$. Let $E:=H_{0}^{1}(\Omega)$ and $W:=H_{0}^{1}(\Omega) \times H_{0}^{1}(\Omega)$, where $H_{0}^{1}(\Omega)$ is the usual Sobolev space with the norm $\|\cdot\|_{E}$ generated by the inner product

$$
\langle u, v\rangle_{E}=\int_{\Omega} \nabla u \nabla v d x, \quad u, v \in H_{0}^{1}(\Omega) .
$$


Then for $U=\left(u_{1}, u_{2}\right)$ and $V=\left(v_{1}, v_{2}\right)$ in $W$, the induced inner product and norm on $W$ are given, respectively, by

$$
\langle U, V\rangle_{W}=\left\langle u_{1}, v_{1}\right\rangle_{E}+\left\langle u_{2}, v_{2}\right\rangle_{E} \quad \text { and } \quad\|U\|_{W}^{2}=\left\|u_{1}\right\|_{E}^{2}+\left\|u_{2}\right\|_{E}^{2} .
$$

Let $\vec{e}_{1}:=(1,0)$ and $\vec{e}_{2}:=(0,1)$, then $A^{*} \vec{e}_{1}=\xi \vec{e}_{1}$ and $A^{*} \vec{e}_{2}=\zeta \vec{e}_{2}$ and $\vec{e}_{1} \cdot \vec{e}_{2}=0,\left|\vec{e}_{1}\right|=\left|\vec{e}_{2}\right|=1$. For any $\alpha \in \mathbb{R}$, let $H_{\alpha}^{+}, H_{\alpha}^{-}, H_{\alpha}^{0}$ be the subspaces of $H_{0}^{1}(\Omega)$, where the quadratic form $u \rightarrow\|u\|^{2}-\alpha\|u\|_{2}^{2}$ is positive definite, negative definite and zero, respectively. Let

$$
W^{0}:=H_{\xi}^{0} \times H_{\zeta}^{0}, \quad W^{+}:=H_{\xi}^{+} \times H_{\zeta}^{+} \quad \text { and } \quad W^{-}:=H_{\xi}^{-} \times H_{\zeta}^{-} .
$$

Obviously, $\left(L_{1}\right)$ implies $W^{0}=\{(0,0)\}$.

Let

$$
A_{1}:=\mathbf{i d}-\xi(-\triangle)^{-1} \text { and } \quad A_{2}:=\mathbf{i d}-\zeta(-\triangle)^{-1}
$$

where id denotes the identity from $H_{0}^{1}(\Omega)$ to $H_{0}^{1}(\Omega)$. We introduce an operator $A=\left(A_{1}, A_{2}\right)$ :

$A: W \rightarrow W$, which is defined by $A U=\left(A_{1} u_{1}, A_{2} u_{2}\right)$ for any $U=\left(u_{1}, u_{2}\right) \in W$.

Then $A$ is a bounded self-adjoint operator from $W$ to $W$. The space $W$ splits as $W=W^{-} \oplus W^{+}$, where $W^{-}$and $W^{+}$are invariant under $A,\left.A\right|_{W^{-}}$is negative, and $\left.A\right|_{W^{+}}$is positive definite. More precisely, there exists a positive constant $C_{0}$ such that

$$
\pm\left\langle A U^{ \pm}, U^{ \pm}\right\rangle_{W} \geq C_{0}\left\|U^{ \pm}\right\|_{W}^{2}, \quad \forall U^{ \pm} \in W^{ \pm}
$$

Here and in what follows, for any $U \in W$, we always denote by $U^{+}$and $U^{-}$the vectors in $W$ with $U=U^{-}+U^{+}, U^{ \pm} \in W^{ \pm}$. We know that $\operatorname{dim} W^{-}$is finite.

For problem (1.1), we consider the following functional:

$$
\Phi(U)=\frac{1}{2}\langle A U, U\rangle_{W}-\int_{\Omega} \widetilde{F}(x, U) d x, \quad U=\left(u_{1}, u_{2}\right) \in W,
$$

where $\widetilde{F}(x, s, t)=F\left(x, s \vec{e}_{1}+t \vec{e}_{2}\right)=F(x, s, t)$. Now, we define an equivalent inner product $\langle\cdot, \cdot\rangle$ and the corresponding norm $\|\cdot\|$ on $W$ given, respectively, by

$$
\langle U, V\rangle=\left\langle A U^{+}, V^{+}\right\rangle_{W}-\left\langle A U^{-}, V^{-}\right\rangle_{W} \quad \text { and } \quad\|U\|=\langle U, U\rangle^{\frac{1}{2}},
$$

where $U^{ \pm}, V^{ \pm} \in W^{ \pm}$. Therefore, $\Phi$ can be rewritten as

$$
\Phi(U)=\frac{1}{2}\left\|U^{+}\right\|^{2}-\frac{1}{2}\left\|U^{-}\right\|^{2}-\int_{\Omega} F(x, U) d x .
$$

By our assumptions, it is easy to see that for any $U, V \in W$,

$$
\Phi^{\prime}(U) V=\left\langle U^{+}, V^{+}\right\rangle-\left\langle U^{-}, V^{-}\right\rangle-\int_{\Omega}(\nabla F(x, U), V) d x
$$

and, by the discussion of [3], the (weak) solutions of system (1.1) are the critical points of the $C^{1}$ functional $\Phi: W \rightarrow \mathbb{R}$.

The following abstract critical point theorem plays an important role in proving our main result. Let $W$ be a Hilbert space with norm $\|\cdot\|$ and have 
an orthogonal decomposition $W=N \oplus N^{\perp}, N \subset W$ is a closed and separable subspace. There exists norm $|V|_{\omega}$ satisfies $|V|_{\omega} \leq\|V\|$ for all $V \in N$ and induces an topology equivalent to the weak topology of $N$ on bounded subset of $N$. For $U=V+Z \in W=N \oplus N^{\perp}$ with $V \in N, Z \in N^{\perp}$, we define $|U|_{\omega}^{2}=|V|_{\omega}^{2}+\|Z\|^{2}$. Particularly, if $\left(U_{n}=V_{n}+Z_{n}\right)$ is $\|\cdot\|$-bounded and

$U_{n} \stackrel{|\cdot|_{\omega}}{\rightarrow} U$, then $V_{n} \rightarrow V$ weakly in $N, Z_{n} \rightarrow Z$ strongly in $N^{\perp}, U_{n} \rightarrow V+Z$ weakly in $W$ (cf. [15]).

Let $W=W^{-} \oplus W^{+}, Z_{0} \in W^{+}$with $\left\|Z_{0}\right\|=1$. Let $N:=W^{-} \oplus \mathbb{R} Z_{0}$ and $W_{1}^{+}:=N^{\perp}=\left(W^{-} \oplus \mathbb{R} Z_{0}\right)^{\perp}$. For $R>0$, let

$$
Q:=\left\{U:=U^{-}+s Z_{0}: s \in \mathbb{R}^{+}, U^{-} \in W^{-},\|U\|<R\right\}
$$

with $P_{0}=s_{0} Z_{0} \in Q, s_{0}>0$. We define

$$
D:=\left\{U:=s Z_{0}+Z^{+}: s \in \mathbb{R}, Z^{+} \in W_{1}^{+},\left\|s Z_{0}+Z^{+}\right\|=s_{0}\right\} .
$$

For $\Phi \in C^{1}(W, \mathbb{R})$, we define

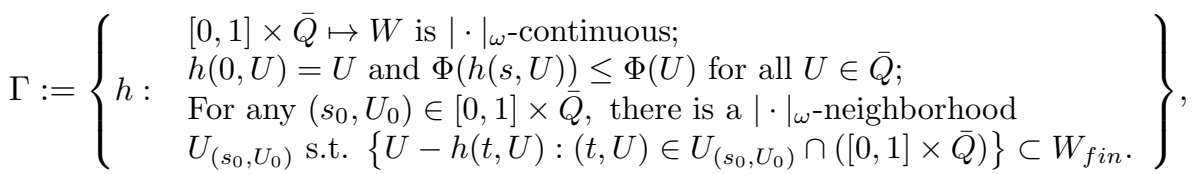

where $W_{\text {fin }}$ denotes various finite-dimensional subspaces of $W, \Gamma \neq 0$ since $i d \in \Gamma$.

The variant weak linking theorem is:

Lemma 2.1 ([15]). The family of $C^{1}$-functional $\left\{\Phi_{\lambda}\right\}$ has the form

$$
\Phi_{\lambda}(U):=J(U)-\lambda K(U), \quad \forall \lambda \in[1,2] .
$$

Assume that

(a) $K(U) \geq 0, \forall U \in W, \Phi_{1}=\Phi$;

(b) $J(U) \rightarrow \infty$ or $K(U) \rightarrow \infty$ as $\|U\| \rightarrow \infty$;

(c) $\Phi_{\lambda}$ is $|\cdot|{ }_{\omega}$-upper semicontinuous, $\Phi_{\lambda}^{\prime}$ is weakly sequentially continuous on $W$. Moreover, $\Phi_{\lambda}$ maps bounded sets to bounded sets;

(d) $\sup _{\partial Q} \Phi_{\lambda}<\inf _{D} \Phi_{\lambda}, \forall \lambda \in[1,2]$.

Then for almost all $\lambda \in[1,2]$, there exists a sequence $\left\{U_{n}\right\}$ such that

$$
\sup _{n}\left\|U_{n}\right\|<\infty, \quad \Phi_{\lambda}^{\prime}\left(U_{n}\right) \rightarrow 0, \quad \Phi_{\lambda}\left(U_{n}\right) \rightarrow c_{\lambda}
$$

where $c_{\lambda}:=\inf _{h \in \Gamma} \sup _{U \in Q} \Phi_{\lambda}(h(1, U)) \in\left[\inf _{D} \Phi_{\lambda}, \sup _{\bar{Q}} \Phi\right]$.

In order to apply Lemma 2.1 , we consider

$$
\Phi_{\lambda}(U):=\frac{1}{2}\left\|U^{+}\right\|^{2}-\lambda\left(\frac{1}{2}\left\|U^{-}\right\|^{2}+\int_{\Omega} F(x, U) d x\right) .
$$

It is easy to see that $\Phi_{\lambda}$ satisfies conditions (a), (b) in Lemma 2.1. To see (c), if $U_{n} \stackrel{|\cdot|_{\omega}}{\rightarrow} U$, then $U_{n}^{+} \rightarrow U^{+}$and $U_{n}^{-} \rightarrow U^{-}$in $W$, going to a subsequence 
if necessary, $U_{n} \rightarrow U$ a.e. on $\Omega$. Using Fatou's lemma and the weak lower semicontinuity of the norm, we have

$$
\varlimsup_{n \rightarrow \infty} \Phi_{\lambda}\left(U_{n}\right) \leq \Phi_{\lambda}(U),
$$

which means that $\Phi_{\lambda}$ is $|\cdot|_{\omega}$-upper semicontinuous. $\Phi_{\lambda}^{\prime}$ is weakly sequentially continuous on $W$ is due to [18]. To continue the discussion, we still need to verify condition $(d)$.

Lemma 2.2. Under the assumptions of Theorem 1.1, we have the following facts:

(i) There exists $\rho>0$ independent of $\lambda \in[1,2]$ such that $\kappa:=\inf \Phi_{\lambda}\left(S_{\rho} W^{+}\right)$ $>0$, where

$$
S_{\rho} W^{+}:=\left\{Z \in W^{+}:\|Z\|=\rho\right\} .
$$

(ii) For fixed $Z_{0} \in W^{+}$with $\left\|Z_{0}\right\|=1$ and any $\lambda \in[1,2]$, there is $R>\rho>0$ such that $\sup \Phi_{\lambda}(\partial Q) \leq 0$, where $Q:=\left\{U:=U^{-}+s Z_{0}: s \in \mathbb{R}^{+}, U^{-} \in W^{-}\right.$, $\|U\|<R\}$.

Proof. (i) Under assumptions $\left(S_{1}\right)$ and $\left(S_{4}\right)$, we know for any $\varepsilon>0$ there exists $C_{\varepsilon}>0$ such that

$$
|\nabla F(x, U)| \leq \varepsilon|U|+C_{\varepsilon}|U|^{p-1}
$$

and

$$
|F(x, U)| \leq \varepsilon|U|^{2}+C_{\varepsilon}|U|^{p},
$$

where $p \geq \frac{2 \sigma}{\sigma-1}>2$ with $\sigma>1$. Hence, for any $U \in W^{+}$,

$$
\Phi_{\lambda}(U) \geq \frac{1}{2}\|U\|^{2}-\lambda \varepsilon\|U\|^{2}-C_{\varepsilon}^{\prime}\|U\|^{p}
$$

which implies the conclusion.

(ii) Suppose by contradiction that there exist $U_{n} \in W^{-} \oplus \mathbb{R}^{+} Z_{0}$ such that $\Phi_{\lambda}\left(U_{n}\right)>0$ for all $n$ and $\left\|U_{n}\right\| \rightarrow \infty$ as $n \rightarrow \infty$. Let $V_{n}:=\frac{U_{n}}{\left\|U_{n}\right\|}=s_{n} Z_{0}+V_{n}^{-}$, then

$$
0<\frac{\Phi_{\lambda}\left(U_{n}\right)}{\left\|U_{n}\right\|^{2}}=\frac{1}{2}\left(s_{n}^{2}-\lambda\left\|V_{n}^{-}\right\|^{2}\right)-\lambda \int_{\Omega} \frac{F\left(x, U_{n}\right)}{U_{n}^{2}} V_{n}^{2} d x .
$$

From $\left(S_{3}\right)$, we know $F(x, U) \geq 0$ and have

$$
\left\|V_{n}^{-}\right\|^{2} \leq \lambda\left\|V_{n}^{-}\right\|^{2}<s_{n}^{2}=1-\left\|V_{n}^{-}\right\|^{2},
$$

therefore, $\left\|V_{n}^{-}\right\| \leq \frac{1}{\sqrt{2}}$ and $1-\frac{1}{\sqrt{2}} \leq s_{n} \leq 1$.

Thus $s_{n} \rightarrow s \neq 0$ after passing to a subsequence, $V_{n} \rightarrow V$ and $V_{n} \rightarrow V$ a.e. on $\Omega$. Hence, $V=s Z_{0}+V^{-} \neq 0$ and, since $\left|U_{n}\right| \rightarrow \infty$ if $V \neq 0$, it follows from $\left(S_{2}\right)$ and Fatou's lemma that

$$
\int_{\Omega} \frac{F\left(x, U_{n}\right)}{U_{n}^{2}} V_{n}^{2} d x \rightarrow+\infty
$$

contrary to (2.5). The proof is finished. 
Applying Lemma 2.1, we soon obtain the following facts:

Lemma 2.3. Under the assumptions of Theorem 1.1, for almost all $\lambda \in[1,2]$, there exists a sequence $\left\{U_{n}\right\}$ such that

$$
\sup _{n}\left\|U_{n}\right\|<\infty, \quad \Phi_{\lambda}^{\prime}\left(U_{n}\right) \rightarrow 0, \quad \Phi_{\lambda}\left(U_{n}\right) \rightarrow c_{\lambda} \in\left[\kappa, \sup _{\bar{Q}} \Phi\right] .
$$

Lemma 2.4. Under the assumptions of Theorem 1.1, for almost all $\lambda \in[1,2]$, there exists a $U_{\lambda}$ such that

$$
\Phi_{\lambda}^{\prime}\left(U_{\lambda}\right)=0, \quad \Phi_{\lambda}\left(U_{\lambda}\right) \leq \sup _{\bar{Q}} \Phi .
$$

Proof. Let $\left\{U_{n}\right\}$ be the sequence obtained in Lemma 2.3, write $U_{n}=U_{n}^{-}+U_{n}^{+}$ with $U_{n}^{ \pm} \in W^{ \pm}$. Since $\left\{U_{n}\right\}$ is bounded, $\left\{U_{n}^{+}\right\}$is also bounded, then $U_{n} \rightarrow U_{\lambda}$ and $U_{n}^{+} \rightarrow U_{\lambda}^{+}$in $W$, after passing to a subsequence. The Sobolev compactly embedding theorem implies $U_{n}^{+} \rightarrow U_{\lambda}^{+}$in $L^{p}(\Omega) \times L^{p}(\Omega)$ for all $p \in\left[2,2^{*}\right)$, where $2^{*}:=\frac{2 N}{N-2}$ if $N \geq 3$ and $2^{*}:=+\infty$ if $N=1,2$.

We claim that $U_{\lambda}^{+} \neq 0$. If not, then $U_{n}^{+} \rightarrow 0$ in $L^{p}(\Omega) \times L^{p}(\Omega)$ for all $p \in\left[2,2^{*}\right)$. It follows from the definition of $\Phi$, Hölder's inequality and (2.3) that

$$
\begin{aligned}
0 \leq \int_{\Omega}\left|\left(\nabla F\left(x, U_{n}\right), U_{n}^{+}\right)\right| d x & \leq \varepsilon \int_{\Omega}\left|U_{n}\right| \cdot\left|U_{n}^{+}\right| d x+C_{\varepsilon} \int_{\Omega}\left|U_{n}\right|^{p-1}\left|U_{n}^{+}\right| d x \\
& \leq \varepsilon\left\|U_{n}\right\|_{2}\left\|U_{n}^{+}\right\|_{2}+C_{\varepsilon}\left\|U_{n}\right\|_{p}^{p-1}\left\|U_{n}^{+}\right\|_{p} \rightarrow 0 .
\end{aligned}
$$

Therefore,

$$
\Phi_{\lambda}\left(U_{n}\right) \leq\left\|U_{n}^{+}\right\|^{2}=\Phi_{\lambda}^{\prime}\left(U_{n}\right) U_{n}^{+}+\lambda \int_{\Omega}\left(\nabla F\left(x, U_{n}\right), U_{n}^{+}\right) d x \rightarrow 0,
$$

which contradicts with the fact that $\Phi_{\lambda}\left(U_{n}\right) \geq \kappa$. Hence, $U_{\lambda}^{+} \neq 0$, and thus $U_{\lambda} \neq 0$. Note that $\Phi_{\lambda}^{\prime}$ is weakly sequentially continuous on $W$, thus

$$
\Phi_{\lambda}^{\prime}\left(U_{\lambda}\right) Z=\lim _{n \rightarrow \infty} \Phi_{\lambda}^{\prime}\left(U_{n}\right) Z=0, \quad \forall Z \in W .
$$

By $\left(S_{3}\right)$, Fatou's lemma and Lemma 2.3, we have

$$
\begin{aligned}
\sup _{\bar{Q}} \Phi \geq c_{\lambda} & =\lim _{n \rightarrow \infty}\left(\Phi_{\lambda}\left(U_{n}\right)-\frac{1}{2} \Phi_{\lambda}^{\prime}\left(U_{n}\right) U_{n}\right) \\
& =\lim _{n \rightarrow \infty} \lambda \int_{\Omega}\left(\frac{1}{2}\left(\nabla F\left(x, U_{n}\right), U_{n}\right)-F\left(x, U_{n}\right)\right) d x \\
& \geq \lambda \int_{\Omega}\left(\frac{1}{2}\left(\nabla F\left(x, U_{\lambda}\right), U_{\lambda}\right)-F\left(x, U_{\lambda}\right)\right) d x=\Phi_{\lambda}\left(U_{\lambda}\right) .
\end{aligned}
$$

Thus we get $\Phi_{\lambda}\left(U_{\lambda}\right) \leq \sup _{\bar{Q}} \Phi$.

Lemma 2.5. Under the assumptions of Theorem 1.1, there exists $\lambda_{n} \rightarrow 1$ and $\left\{U_{\lambda_{n}}\right\}$ such that

$$
\Phi_{\lambda_{n}}^{\prime}\left(U_{\lambda_{n}}\right)=0, \quad \Phi_{\lambda_{n}}\left(U_{\lambda_{n}}\right) \leq \sup _{\bar{Q}} \Phi
$$


Moreover, $\left\{U_{\lambda_{n}}\right\}$ is bounded.

Proof. The existence of $\left\{U_{\lambda_{n}}\right\}$ such that

$$
\Phi_{\lambda_{n}}^{\prime}\left(U_{\lambda_{n}}\right)=0, \quad \Phi_{\lambda_{n}}\left(U_{\lambda_{n}}\right) \leq \sup _{\bar{Q}} \Phi
$$

is the direct consequence of Lemma 2.4. To prove the boundedness of $\left\{U_{\lambda_{n}}\right\}$, arguing by contradiction, suppose that $\left\|U_{\lambda_{n}}\right\| \rightarrow \infty$. Let $V_{\lambda_{n}}:=\frac{U_{\lambda_{n}}}{\left\|U_{\lambda_{n}}\right\|}$. Then $\left\|V_{\lambda_{n}}\right\|=1, V_{\lambda_{n}} \rightarrow V$ in $W$ and $V_{\lambda_{n}} \rightarrow V$ a.e. in $\Omega$, after passing to a subsequence.

Recall that $\Phi_{\lambda_{n}}^{\prime}\left(U_{\lambda_{n}}\right)=0$. Thus for any $\varphi \in W$, we have

$$
\left\langle U_{\lambda_{n}}^{+}, \varphi\right\rangle-\lambda_{n}\left\langle U_{\lambda_{n}}^{-}, \varphi\right\rangle=\lambda_{n} \int_{\Omega}\left(\nabla F\left(x, U_{\lambda_{n}}\right), \varphi\right) d x .
$$

Consequently $\left\{V_{\lambda_{n}}\right\}$ satisfies

$$
\left\langle V_{\lambda_{n}}^{+}, \varphi\right\rangle-\lambda_{n}\left\langle V_{\lambda_{n}}^{-}, \varphi\right\rangle=\lambda_{n} \int_{\Omega} \frac{\left(\nabla F\left(x, U_{\lambda_{n}}\right), \varphi\right)}{\left\|U_{\lambda_{n}}\right\|} d x .
$$

Let $\varphi=V_{\lambda_{n}}^{ \pm}$in (2.7), respectively. Then we have

$$
\left\langle V_{\lambda_{n}}^{+}, V_{\lambda_{n}}^{+}\right\rangle=\lambda_{n} \int_{\Omega} \frac{\left(\nabla F\left(x, U_{\lambda_{n}}\right), V_{\lambda_{n}}^{+}\right)}{\left\|U_{\lambda_{n}}\right\|} d x
$$

and

$$
-\lambda_{n}\left\langle V_{\lambda_{n}}^{-}, V_{\lambda_{n}}^{-}\right\rangle=\lambda_{n} \int_{\Omega} \frac{\left(\nabla F\left(x, U_{\lambda_{n}}\right), V_{\lambda_{n}}^{-}\right)}{\left\|U_{\lambda_{n}}\right\|} d x .
$$

Since $1=\left\|V_{\lambda_{n}}\right\|^{2}=\left\|V_{\lambda_{n}}^{+}\right\|^{2}+\left\|V_{\lambda_{n}}^{-}\right\|^{2}$, we have

$$
1=\int_{\Omega} \frac{\left(\nabla F\left(x, U_{\lambda_{n}}\right), \lambda_{n} V_{\lambda_{n}}^{+}-V_{\lambda_{n}}^{-}\right)}{\left\|U_{\lambda_{n}}\right\|} d x .
$$

For $r \geq 0$, let

$$
h(r):=\inf \left\{\widetilde{F}(x, U): x \in \Omega \text { and } U \in \mathbb{R}^{2} \text { with }|U| \geq r\right\} .
$$

By $\left(S_{3}\right)$, we have $h(r)>0$ for all $r>0$. By $\left(S_{3}\right)$ and $\left(S_{4}\right)$, for $|U| \geq r_{0}$,

$$
\begin{aligned}
c_{0} \widetilde{F}(x, U) \geq \frac{|\nabla F(x, U)|^{\sigma}}{\mid U^{\sigma}} & =\left(\frac{|\nabla F(x, U)||U|}{|U|^{2}}\right)^{\sigma} \\
& \geq\left(\frac{(\nabla F(x, U), U)}{|U|^{2}}\right)^{\sigma} \geq\left(\frac{2 F(x, U)}{|U|^{2}}\right)^{\sigma},
\end{aligned}
$$

it follows form $\left(S_{2}\right)$ and the definition of $h(r)$ that

$$
h(r) \rightarrow \infty \quad \text { as } r \rightarrow \infty .
$$

For $0<a<b$, let

$$
\Omega_{n}(a, b):=\left\{x \in \Omega: a \leq\left|U_{\lambda_{n}}(x)\right|<b\right\}
$$


and

$$
C_{a}^{b}:=\inf \left\{\frac{\widetilde{F}(x, U)}{|U|^{2}}: x \in \Omega \text { and } U \in \mathbb{R}^{2} \text { with } a \leq|U| \leq b\right\} .
$$

Since $F(x, U)$ depends periodically on $x$ and $\widetilde{F}(x, U)>0$ if $U \in \mathbb{R}^{2} \backslash\{(0,0)\}$, one has $C_{a}^{b}>0$ and

$$
\widetilde{F}\left(x, U_{\lambda_{n}}\right) \geq C_{a}^{b}\left|U_{\lambda_{n}}\right|^{2} \quad \text { for all } x \in \Omega_{n}(a, b) .
$$

Since $\Phi_{\lambda_{n}}^{\prime}\left(U_{\lambda_{n}}\right)=0$ and $\Phi_{\lambda_{n}}\left(U_{\lambda_{n}}\right) \leq \sup _{\bar{Q}} \Phi$, there exists a constant $C_{0}>0$ such that for all $n$

$$
C_{0} \geq \Phi_{\lambda_{n}}\left(U_{\lambda_{n}}\right)-\frac{1}{2} \Phi_{\lambda_{n}}^{\prime}\left(U_{\lambda_{n}}\right) U_{\lambda_{n}}=\int_{\Omega} \widetilde{F}\left(x, U_{\lambda_{n}}\right) d x
$$

from which we have

$$
\begin{aligned}
C_{0} & \geq \int_{\Omega_{n}(0, a)} \widetilde{F}\left(x, U_{\lambda_{n}}\right) d x+\int_{\Omega_{n}(a, b)} \tilde{F}\left(x, U_{\lambda_{n}}\right) d x+\int_{\Omega_{n}(b, \infty)} \tilde{F}\left(x, U_{\lambda_{n}}\right) d x \\
& \geq \int_{\Omega_{n}(0, a)} \widetilde{F}\left(x, U_{\lambda_{n}}\right) d t+C_{a}^{b} \int_{\Omega_{n}(a, b)}\left|U_{\lambda_{n}}\right|^{2} d x+h(b)\left|\Omega_{n}(b, \infty)\right| .
\end{aligned}
$$

Invoking $\left(S_{4}\right)$, set $\tau:=2 \sigma /(\sigma-1)$ and $\sigma^{\prime}=\tau / 2$. Since $\sigma>\max \{1, N / 2\}$ one sees $\tau \in\left(2,2^{*}\right)$. Fix arbitrarily $\hat{\tau} \in\left(\tau, 2^{*}\right)$. Using (2.10), we have

$$
\left|\Omega_{n}(b, \infty)\right| \leq \frac{C_{0}}{h(b)} \rightarrow 0
$$

as $b \rightarrow \infty$ uniformly in $n$, it follows from $\left\|V_{\lambda_{n}}\right\|=1$, the Hölder inequality and the Sobolev embedding theorem that

$$
\int_{\Omega_{n}(b, \infty)}\left|V_{\lambda_{n}}\right|^{\tau} d x \leq C\left|\Omega_{n}(b, \infty)\right|^{1-\frac{\tau}{\hat{\tau}}} \rightarrow 0
$$

as $b \rightarrow \infty$ uniformly in $n$. Using (2.10) again, for any fixed $0<a<b$,

$$
\int_{\Omega_{n}(a, b)}\left|V_{\lambda_{n}}\right|^{2} d x=\frac{1}{\left\|U_{\lambda_{n}}\right\|^{2}} \int_{\Omega_{n}(a, b)}\left|U_{\lambda_{n}}\right|^{2} d x \leq \frac{C_{0}}{C_{a}^{b}\left\|U_{\lambda_{n}}\right\|^{2}} \rightarrow 0 \quad \text { as } n \rightarrow \infty .
$$

Let $0<\varepsilon<\frac{1}{3}$. Sobolev embedding theorem implies $\left\|V_{\lambda_{n}}\right\|_{2}^{2} \leq C\left\|V_{\lambda_{n}}\right\|^{2}=C$ and $\left|\lambda_{n}\right| \leq C_{1}$. By $\left(S_{1}\right)$ there is $a_{\varepsilon}>0$ such that $|\nabla F(x, U)|<\frac{\varepsilon}{C_{1} C}|U|$ for all $|U| \leq a_{\varepsilon}$, consequently,

$$
\begin{aligned}
& \int_{\Omega_{n}\left(0, a_{\varepsilon}\right)} \frac{\left(\nabla F\left(x, U_{\lambda_{n}}\right), \lambda_{n} V_{\lambda_{n}}^{+}-V_{\lambda_{n}}^{-}\right)}{|| U_{\lambda_{n}} \mid} d x \\
\leq & \int_{\Omega_{n}\left(0, a_{\varepsilon}\right)} \frac{\left|\nabla F\left(x, U_{\lambda_{n}}\right)\right|}{\left|U_{\lambda_{n}}\right|}\left|V_{\lambda_{n}}\right| \cdot\left|\lambda_{n} V_{\lambda_{n}}^{+}-V_{\lambda_{n}}^{-}\right| d x \\
\leq & \frac{\varepsilon}{C_{1} C} \int_{\Omega_{n}\left(0, a_{\varepsilon}\right)}\left|V_{\lambda_{n}}\right| \cdot\left|\lambda_{n} V_{\lambda_{n}}^{+}-V_{\lambda_{n}}^{-}\right| d x
\end{aligned}
$$




$$
\begin{aligned}
& \leq \frac{\varepsilon}{C_{1} C}\left(\int_{\Omega}\left|V_{\lambda_{n}}\right|^{2} d x\right)^{1 / 2}\left(\int_{\Omega}\left|\lambda_{n} V_{\lambda_{n}}^{+}-V_{\lambda_{n}}^{-}\right|^{2} d x\right)^{1 / 2} \\
& \leq \frac{\varepsilon}{C}\left\|V_{\lambda_{n}}\right\|_{2}^{2} \leq \varepsilon
\end{aligned}
$$

for all $n$. By $\left(S_{4}\right),(2.9)$ and (2.11), we can take $b_{\varepsilon} \geq r_{0}$ large so that

$$
\begin{aligned}
& \int_{\Omega_{n}\left(b_{\varepsilon}, \infty\right)} \frac{\left(\nabla F\left(x, U_{\lambda_{n}}\right), \lambda_{n} V_{\lambda_{n}}^{+}-V_{\lambda_{n}}^{-}\right)}{\| U_{\lambda_{n}} \mid} d x \\
\leq & \int_{\Omega_{n}\left(b_{\varepsilon}, \infty\right)} \frac{\left|\nabla F\left(x, U_{\lambda_{n}}\right)\right|}{\left|U_{\lambda_{n}}\right|}\left|V_{\lambda_{n}}\right| \cdot\left|\lambda_{n} V_{\lambda_{n}}^{+}-V_{\lambda_{n}}^{-}\right| d x \\
\leq & \left(\int_{\Omega_{n}\left(b_{\varepsilon}, \infty\right)} \frac{\left|\nabla F\left(x, U_{\lambda_{n}}\right)\right|^{\sigma}}{\left|U_{\lambda_{n}}\right|^{\sigma}} d x\right)^{1 / \sigma}\left(\int_{\Omega_{n}\left(b_{\varepsilon}, \infty\right)}\left(\left|V_{\lambda_{n}}\right| \cdot\left|\lambda_{n} V_{\lambda_{n}}^{+}-V_{\lambda_{n}}^{-}\right|\right)^{\sigma^{\prime}} d x\right)^{1 / \sigma^{\prime}} \\
\leq & \left(\int_{\Omega} c_{0} \widetilde{F}\left(x, U_{\lambda_{n}}\right) d x\right)^{1 / \sigma}\left(\int_{\Omega}\left|\lambda_{n} V_{\lambda_{n}}^{+}-V_{\lambda_{n}}^{-}\right|^{\tau} d x\right)^{1 / \tau}\left(\int_{\Omega_{n}\left(b_{\varepsilon}, \infty\right)}\left|V_{\lambda_{n}}\right|^{\tau} d x\right)^{1 / \tau} \\
< & \varepsilon
\end{aligned}
$$

for all $n$. Note that there is $\gamma=\gamma(\varepsilon)>0$ independent of $n$ such that $\left|\nabla F\left(x, U_{\lambda_{n}}\right)\right| \leq \gamma\left|U_{\lambda_{n}}\right|$ for $t \in \Omega_{n}\left(a_{\varepsilon}, b_{\varepsilon}\right)$. By (2.12) there is $n_{0}$ such that

$$
\begin{aligned}
& \int_{\Omega_{n}\left(a_{\varepsilon}, b_{\varepsilon}\right)} \frac{\left(\nabla F\left(x, U_{\lambda_{n}}\right), \lambda_{n} V_{\lambda_{n}}^{+}-V_{\lambda_{n}}^{-}\right)}{\| U_{\lambda_{n}} \mid} d x \\
\leq & \int_{\Omega_{n}\left(a_{\varepsilon}, b_{\varepsilon}\right)} \frac{\left|\nabla F\left(x, U_{\lambda_{n}}\right)\right|}{\left|U_{\lambda_{n}}\right|}\left|V_{\lambda_{n}}\right| \cdot\left|\lambda_{n} V_{\lambda_{n}}^{+}-V_{\lambda_{n}}^{-}\right| d x \\
\leq & \gamma \int_{\Omega_{n}\left(a_{\varepsilon}, b_{\varepsilon}\right)}\left|V_{\lambda_{n}}\right| \cdot\left|\lambda_{n} V_{\lambda_{n}}^{+}-V_{\lambda_{n}}^{-}\right| d x \\
\leq & \gamma\left(\int_{\Omega}\left|V_{\lambda_{n}}\right|^{2} d t\right)^{1 / 2}\left(\int_{\Omega_{n}\left(a_{\varepsilon}, b_{\varepsilon}\right)}\left|\lambda_{n} V_{\lambda_{n}}^{+}-V_{\lambda_{n}}^{-}\right|^{2} d x\right)^{1 / 2} \\
\leq & \gamma \lambda_{n}\left\|V_{\lambda_{n}}\right\|_{2}\left(\int_{\Omega_{n}\left(a_{\varepsilon}, b_{\varepsilon}\right)}\left|V_{\lambda_{n}}\right|^{2} d x\right)^{1 / 2}<\varepsilon
\end{aligned}
$$

for all $n \geq n_{0}$. Therefore, the combination of (2.13)-(2.15) implies that for $n \geq n_{0}$, we have

$$
\int_{\Omega} \frac{\left(\nabla F\left(x, U_{\lambda_{n}}\right), \lambda_{n} V_{\lambda_{n}}^{+}-V_{\lambda_{n}}^{-}\right)}{\left\|U_{\lambda_{n}}\right\|} d x<3 \varepsilon<1,
$$

which contradicts with (2.8). Thus $\left\{U_{\lambda_{n}}\right\}$ is bounded. 
Lemma 2.6. If $\left\{U_{\lambda_{n}}\right\}$ is the sequence obtained in Lemma 2.5, then it is also a (PS) sequence for $\Phi$ satisfying

$$
\lim _{n \rightarrow \infty} \Phi^{\prime}\left(U_{\lambda_{n}}\right)=0, \quad \lim _{n \rightarrow \infty} \Phi\left(U_{\lambda_{n}}\right) \leq \sup _{\bar{Q}} \Phi .
$$

Proof. Note that $U_{\lambda_{n}}$ is bounded. From

$$
\lim _{n \rightarrow \infty} \Phi\left(U_{\lambda_{n}}\right)=\lim _{n \rightarrow \infty}\left[\Phi_{\lambda_{n}}\left(U_{\lambda_{n}}\right)+\left(\lambda_{n}-1\right)\left(\frac{1}{2}\left\|U_{\lambda_{n}}^{-}\right\|^{2}+\int_{\Omega} F\left(x, U_{\lambda_{n}}\right) d x\right)\right]
$$

and note that

$$
\begin{aligned}
& \lim _{n \rightarrow \infty} \Phi^{\prime}\left(U_{\lambda_{n}}\right) \varphi \\
= & \lim _{n \rightarrow \infty}\left[\Phi_{\lambda_{n}}^{\prime}\left(U_{\lambda_{n}}\right) \varphi+\left(\lambda_{n}-1\right)\left(\left\langle U_{\lambda_{n}}^{-}, \varphi^{-}\right\rangle+\int_{\Omega}\left(\nabla F\left(x, U_{\lambda_{n}}\right), \varphi\right) d x\right)\right]
\end{aligned}
$$

uniformly in $\varphi \in W$, we obtain the conclusion.

\section{Proof of main result}

We are now in a position to prove our main result.

Proof of Theorem 1.1. Note that Lemma 2.5 implies $\left\{U_{\lambda_{n}}\right\}$ is bounded, thus $U_{\lambda_{n}} \rightarrow U$ in $W$, after passing to a subsequence. The Sobolev compactly embedding theorem implies $U_{\lambda_{n}} \rightarrow U$ in $L^{q}(\Omega) \times L^{q}(\Omega)$ for all $q \in\left[2,2^{*}\right)$, where $2^{*}:=\frac{2 N}{N-2}$ if $N \geq 3$ and $2^{*}:=+\infty$ if $N=1,2$.

By $\Phi_{\lambda_{n}}^{\prime}\left(U_{\lambda_{n}}\right) U_{\lambda_{n}}^{+}=0$, Hölder's inequality, Sobolev embedding theorem and (2.3), we know

$$
\begin{aligned}
\left\|U_{\lambda_{n}}^{+}\right\|^{2} & =\lambda \int_{\Omega}\left(\nabla F\left(x, U_{\lambda_{n}}\right), U_{\lambda_{n}}^{+}\right) d x \\
& \leq \varepsilon \int_{\Omega}\left|U_{\lambda_{n}}\right| \cdot\left|U_{\lambda_{n}}^{+}\right| d x+C_{\varepsilon} \int_{\Omega}\left|U_{\lambda_{n}}\right|^{p-1}\left|U_{\lambda_{n}}^{+}\right| d x \\
& \leq \varepsilon\left\|U_{\lambda_{n}}\right\| \cdot\left\|U_{\lambda_{n}}^{+}\right\|+C_{\varepsilon}^{\prime}\left\|U_{\lambda_{n}}\right\|_{p}^{p-1}\left\|U_{\lambda_{n}}^{+}\right\| \\
& \leq \varepsilon\left\|U_{\lambda_{n}}\right\| \cdot\left\|U_{\lambda_{n}}^{+}\right\|+C_{\varepsilon}^{\prime \prime}\left\|U_{\lambda_{n}}\right\|_{p}^{p-2}\left\|U_{\lambda_{n}}\right\| \cdot\left\|U_{\lambda_{n}}^{+}\right\| \\
& \leq \varepsilon\left\|U_{\lambda_{n}}\right\|^{2}+C_{\varepsilon}^{\prime \prime}\left\|U_{\lambda_{n}}\right\|_{p}^{p-2}\left\|U_{\lambda_{n}}\right\|^{2} .
\end{aligned}
$$

Similarly, we have

$$
\left\|U_{\lambda_{n}}^{-}\right\|^{2} \leq \varepsilon\left\|U_{\lambda_{n}}\right\|^{2}+C_{\varepsilon}^{\prime \prime}\left\|U_{\lambda_{n}}\right\|_{p}^{p-2}\left\|U_{\lambda_{n}}\right\|^{2} .
$$

From (3.1) and (3.2), we get

$$
\left\|U_{\lambda_{n}}\right\|^{2} \leq 2 \varepsilon\left\|U_{\lambda_{n}}\right\|^{2}+2 C_{\varepsilon}^{\prime \prime}\left\|U_{\lambda_{n}}\right\|_{p}^{p-2}\left\|U_{\lambda_{n}}\right\|^{2},
$$

which means $\left\|U_{\lambda_{n}}\right\|_{p} \geq c$ for some constant $c$, it follows from $U_{\lambda_{n}} \rightarrow U$ in $L^{p}(\Omega) \times L^{p}(\Omega)$ that $U \neq 0$. The facts that $\Phi_{\lambda}^{\prime}$ is weakly sequentially continuous on $W$ and $U_{\lambda_{n}} \rightarrow U$ in $W$ imply $\Phi^{\prime}(U)=0$. 
Let $K:=\left\{U \in W: \Phi^{\prime}(U)=0\right\}$ be the critical set of $\Phi$ and

$$
C:=\inf \{\Phi(Z): Z \in K \backslash\{0\}\} .
$$

For any critical point $U$ of $\Phi$, assumption $\left(S_{3}\right)$ implies that

$$
\begin{aligned}
\Phi(U) & =\Phi(U)-\frac{1}{2} \Phi^{\prime}(U) U \\
& =\int_{\Omega}\left(\frac{1}{2}(\nabla F(x, U), U)-F(x, U)\right) d x>0 \quad \text { if } U \neq 0 .
\end{aligned}
$$

Therefore $C \geq 0$. We prove that $C>0$ and there is $U \in K$ such that $\Phi(U)=C$. Let $U_{j} \in K \backslash\{0\}$ be such that $\Phi\left(U_{j}\right) \rightarrow C$. Then, the proof in Lemma 2.5 shows that $\left\{U_{j}\right\}$ is bounded, and by the concentration compactness principle discussion above we know $U_{j} \rightarrow U \in K \backslash\{0\}$. Thus

$$
\begin{aligned}
C=\lim _{j \rightarrow \infty} \Phi\left(U_{j}\right) & =\lim _{j \rightarrow \infty} \int_{\Omega}\left(\frac{1}{2}\left(\nabla F\left(x, U_{j}\right), U_{j}\right)-F\left(x, U_{j}\right)\right) d x \\
& \geq \int_{\Omega}\left(\frac{1}{2}(\nabla F(x, U), U)-F(x, U)\right) d x=\Phi(U) \geq C,
\end{aligned}
$$

the first inequality dues to $\left(S_{3}\right)$ and the Fatou's lemma. So $\Phi(U)=C$ and $C>0$ because $U \neq 0$.

\section{References}

[1] G. Chen and S. Ma, Infinitely many solutions for resonant cooperative elliptic systems with sublinear or superlinear terms, Calc. Var. Partial Differential Equations 49 (2014), no. 1-2, 271-286.

[2] _ Asymptotically or super linear cooperative elliptic systems in the whole space, Sci. China Math. 56 (2013), no. 6, 1181-1194.

[3] D. G. Costa and C. A. Magalhães, A variational approach to subquadratic perturbations of elliptic systems, J. Differential Equations 111 (1994), no. 1, 103-122.

[4] _ A unified approach to a class of strongly indefinite functionals, J. Differential Equations 125 (1996), no. 2, 521-547.

[5] G. Fei, Multiple solutions of some nonlinear strongly resonant elliptic equations without the (PS) condition, J. Math. Anal. Appl. 193 (1995), no. 2, 659-670.

[6] M. Lazzo, Nonlinear differential problems and Morse thoery, Nonlinear Anal. 30 (1997), no. $1,169-176$.

[7] S. Li and J. Q. Liu, Computations of critical groups at degenerate critical point and applications to nonlinear differential equations with resonance, Houston J. Math. 25 (1999), no. 3, 563-582.

[8] S. Li and W. Zou, The Computations of the critical groups with an application to elliptic resonant problems at a higher eigenvalue, J. Math. Anal. Appl. 235 (1999), no. 1, 237259 .

[9] S. Ma, Infinitely many solutions for cooperative elliptic systems with odd nonlinearity, Nonlinear Anal. 71 (2009), no. 5-6, 1445-1461.

[10] _ Nontrivial solutions for resonant cooperative elliptic systems via computations of the critical groups, Nonlinear Anal. 73 (2010), no. 12, 3856-3872.

[11] A. Pomponio, Asymptotically linear cooperative elliptic system: existence and multiplicity, Nonlinear Anal. 52 (2003), no. 3, 989-1003. 
[12] P. H. Rabinowitz, Minimax Methods in Critical Point Theory with Applications to Differential Equations, CBMS Reg. Conf. Ser. Math., vol. 65, Amer. Math. Soc., Providence, RI, 1986.

[13] M. Ramos and H. Tavares, Solutions with multiple spike patterns for an elliptic system, Calc. Var. Partial Differential Equations 31 (2008), no. 1, 1-25.

[14] M. Ramos and J. F. Yang, Spike-layered solutions for an elliptic system with Neumann boundary conditions, Trans. Amer. Math. Soc. 357 (2005), no. 8, 3265-3284.

[15] M. Schechter and W. Zou, Weak linking theorems and Schrödinger equations with critical Sobolev exponent, ESAIM Control Optim. Calc. Var. 9 (2003), 601-619.

[16] A. Szulkin and T. Weth, Ground state solutions for some indefinite variational problems, J. Functional Analysis 257 (2009), no. 12, 3802-3822.

[17] C.-L. Tang and Q.-J. Gao, Elliptic resonant problems at higher eigenvalues with an unbounded nonlinear term, J. Differential Equations 146 (1998), no. 1, 56-66.

[18] M. Willem, Minimax Theorems, Birkhäuser, Boston, 1996.

[19] W. Zou, Solutions for resonant elliptic systems with nonodd or odd nonlinearities, J. Math. Anal. Appl. 223 (1998), no. 2, 397-417.

[20] , Multiple solutions for asymptotically linear elliptic systems, J. Math. Anal. Appl. 255 (2001), no. 1, 213-229.

[21] W. Zou and S. Li, Nontrivial Solutions for resonant cooperative elliptic systems via computations of critical groups, Nonlinear Anal. 38 (1999), no. 2, Ser. A: Theory Methods, $229-247$.

[22] _ Infinitely many solutions for Hamiltonian systems, J. Differential Equations 186 (2002), no. 1, 141-164.

School of Mathematics and Statistics

ANyAng Normal University

Anyang 455000, Henan Province, P. R. China

E-mail address: guanweic@163.com 